\title{
Downregulation of microRNA-497 is associated with upregulation of synuclein $\gamma$ in patients with osteosarcoma
}

\author{
LIANG WANG $^{1}$, HONGWEI GAO ${ }^{1}$, NINGJI GONG ${ }^{2}$ and MINGZHI GONG ${ }^{1}$ \\ ${ }^{1}$ Department of Osteological Surgery and ${ }^{2}$ Emergency Department, \\ The Second Hospital of Shandong University, Jinan, Shandong 250033, P.R. China
}

Received January 29, 2015; Accepted November 5, 2015

DOI: $10.3892 /$ etm.2016.3838

\begin{abstract}
The present study aimed to investigate the effects of microRNA (miRNA/miR)-497 expression levels on the expression levels of synuclein $\gamma$ (SNCG) in serum samples, as well as osteosarcoma and lung-metastatic tissue samples, from patients with osteosarcoma. Between December 2010 and August 2013, fasting peripheral blood was collected from 36 patients with osteosarcoma for serum separation. In addition, osteosarcoma and lung metastatic tissues were resected from 15 osteosarcoma patients with lung metastasis by surgery. Bioinformatics was employed to predict the amount miRNA that binds to SNCG. Reverse transcription-quantitative polymerase chain reaction was used to determine the expression levels of SNCG and miR-497, and western blotting was performed to determine protein expression levels. It was observed that SNCG mRNA and protein expression levels were significantly upregulated in osteosarcoma tissues $(\mathrm{P}<0.01)$. Additionally, SNCG mRNA $(\mathrm{P}<0.01)$ and protein $(\mathrm{P}<0.05)$ expression levels were significantly upregulated in the blood of patients with osteosarcoma. SNCG mRNA and protein expression levels were also significantly upregulated in lung metastatic tissues $(\mathrm{P}<0.01)$. miR-497 was significantly downregulated in all three samples; therefore downregulation of miR-497 may lead to the occurrence, development and metastasis of osteosarcoma through the upregulation of SNCG mRNA. In summary, the upregulation of SNCG in blood, osteosarcoma tissue and lung metastatic tissue samples is associated with the dowregulation of miR-497, suggesting that miR-497 may be a potential marker and therapeutic target for the treatment of osteosarcoma.
\end{abstract}

Correspondence to: Dr Mingzhi Gong, Department of Osteological Surgery, The Second Hospital of Shandong University, 247 Beiyuan Street, Jinan, Shandong 250033, P.R. China

E-mail: gmz333@126.com

Key words: microRNA-497, osteosarcoma, synuclein $\gamma$, lung metastasis

\section{Introduction}

Osteosarcoma is a highly malignant primary tumor of the bone. Often occurring in the bones and skeleton or their auxiliary tissues, osteosarcoma displays characteristics of osteoid tissues directly formed by tumor cells (1). The majority of patients with osteosarcoma undergo amputation to remove the affected bone; however, post-amputation five-year survival rates remain low at 5\%-20\% (2). Therefore, patients are administered high-dose chemotherapy, including cisplatin, adriamycin and methotrexate, prior to the operation (3), and radical surgery remains the first-line therapeutic strategy for the treatment of osteosarcoma. With the promotion of radical surgery and high-dose chemotherapy, the five-year survival rates for patients with osteosarcoma have increased to $\sim 70 \%$ (4). The majority of patients with late osteosarcoma present with tumor metastasis, in particular lung metastasis, in addition to multiple organ failure. However, the exact mechanism of osteosarcoma has yet to be elucidated, despite the continual efforts made by researchers (5). The occurrence and development of osteosarcoma is regulated by multiple interconnected pathways involving several types of mRNA and microRNA (miRNA/miR). One study reported that miR-195 was able to inhibit the invasion and migration of osteosarcoma by regulating fatty acid synthase factor (6).

Synuclein $\gamma$ (SNCG), also known as breast cancer specific gene 1 , belongs to the synuclein gene family, which is highly conserved in mammals (7). SNCG was initially isolated from breast cancer (8). Subsequently, a study revealed that SNCG is involved in the occurrence and development of breast cancer (7). In recent years, it has been identified that SNCG is also important in other types of tumors, including breast, gallbladder, pancreatic and uterine cancer (9-13). However, whether SNCG has regulatory effects on the proliferation and migration of osteosarcoma cells has yet to be determined. In the present study, the effects of SNCG on the proliferation and invasion of osteosarcoma, in addition to the regulatory mechanism of miR-497 on SNCG, were investigated.

\section{Materials and methods}

Patients. Between December 2010 and August 2013, a total of 36 patients were diagnosed with osteosarcoma at The Second Hospital of Shandong University (Jinan, China), according to 
the 2002 World Health Organization (WHO) classification standards (14). During this time period at the same hospital, 26 healthy subjects were enrolled as the control group to provide control blood samples. The staging of the disease was determined according to the Enneking staging standard, as follows: Stage I (low-grade malignancy), stage II (high-grade malignancy) and stage III (metastasis) (15). Patients with stage III osteosarcoma (lung metastasis; $n=15$ ) were selected to undergo tissue resection. Only patients with lung metastases at stage III were included in the present study. Patients received resection of osteosarcoma and lung metastases at our hospital, and received no hormones, Chinese traditional drugs, or radiochemotherapy prior to surgery. Additional inclusion and exclusion criteria were set according to the standards outlined by the WHO (14). All procedures were approved by the Ethics Committee of Shandong University (Jinan, China). Written informed consent was obtained from all patients or their families.

Samples. Fasting peripheral blood was collected from all 36 patients in the morning. Serum was separated by density gradient centrifugation of $10-15 \mathrm{ml}$ peripheral blood at $400 \mathrm{x} \mathrm{g}$ for $10 \mathrm{~min}$, and was subsequently stored in liquid nitrogen. Stage III patients with osteosarcoma $(\mathrm{n}=15)$ underwent surgery for osteosarcoma (amputation or limb salvage surgery) and lung metastasis tissue resection, with negative adjacent tissues $(>5 \mathrm{~cm}$ away from tumor foci) used as controls. Tissue samples were stored in liquid nitrogen.

Bioinformatics. Bioinformatics is the predominant method used for the functional analysis of miRNA. A previous study revealed evidence of the regulation of SNCG by one of its upstream mRNAs, miR-15a (16). Using miRanda (www.microrna.org/microrna/getExprForm.do; version 2010), TargetScan (www.targetscan.org/vert_61; version 6.2), PieTar (www.pictar.mdc-berlin.de; version 2007) and BibiServ (www.bibiserv.techfak.uni-bielefield.be/bibi/ Tools.html; version 2013) databases, the miR-15 family genes were screened (miR-15a, miR-15b, miR-16, miR-195, miR-424 and miR-497), and miR-497 was selected for evaluation in the present study, due to it having similar SNCG-binding sites to miR-15a (Fig. 1).

Reverse transcription-quantitative polymerase chain reaction $(R T-q P C R)$. Total RNA was extracted using TRIzol reagent (cat no. 10606ES60; Yeasen, Shanghai, China) and purified using a miRNeasy Serum/Plasma kit (Guangzhou Jianlun Biological Technology Co., Ltd., Guangzhou, China), according to the manufacturer's protocol. The purity of RNA was determined by the absorbance ratio at 260/280 nm using ultraviolet spectrophotometry (NanoDrop ND-1000; Thermo Fisher Scientific, Waltham, MA, USA). Next, cDNA was obtained by reverse transcription (TIANScript RT kit; Tiangen Biotech Co., Ltd., Beijing, China) of $1 \mu \mathrm{g}$ RNA and stored at $-20^{\circ} \mathrm{C}$. RT-PCR was performed using a SuperReal PreMix kit (FP204; Tiangen Biotech Co., Ltd.). The primers for SNCG were as follows: Upstream, 5'-ACACCCACCATG GATGTCTT-3' and downstream, 5'-ACAGTGTTGACGCTG CTCAC- 3 '. The primers for $\beta$-actin were as follows: Upstream, 5'-TGTTTGAGACCTTCAACACCC-3' and downstream,
3' guguuugguaauACACGACGAu 5' hsa-miR-15a

|।|।|।|ा।

137:5' ggccuguccaccUGUGCUGCUg 3' SNCG

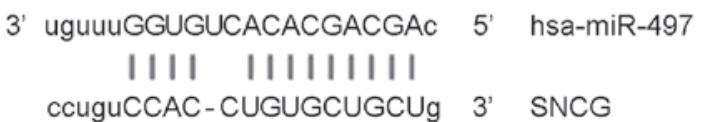

$139: 5^{\prime}$ ccuguCCAC-CUGUGCUGCUg 3' SNCG

Figure 1. Binding sites of miR-15a and miR-497 on SNCG. Using miRanda, TargetScan, PieTar and BibiServ databases, the miR-15 family genes (miR-15a, miR-15b, miR-16, miR-195, miR-424 and miR-497) were screened, and miR-497 was selected for the present study as a result of having similar SNCG-binding sites to miR-15a. miR, microRNA.

5'-AGCACTGTGTGTTGGCGTACAG-3'. PCR amplification was performed on an iQ5 Real-Time PCR Detection System (Bio-Rad Laboratories, Inc. Hercules, CA, USA), as follows: Initial denaturation at $95^{\circ} \mathrm{C}$ for $60 \mathrm{sec} ; 45$ cycles of denaturation at $95^{\circ} \mathrm{C}$ for $5 \mathrm{sec}$, annealing at $60^{\circ} \mathrm{C}$ for $15 \mathrm{sec}$ and elongation at $72^{\circ} \mathrm{C}$ for $5 \mathrm{sec}$. The quantification cycle $(\mathrm{Cq})$ method $\left(2^{-\Delta \Delta \mathrm{Cq}}\right)$ was used to calculate SNCG/ $\beta$-actin expression ratio (17).

miR-497 was isolated from the total RNA using a miRcute miRNA Isolation kit (Tiangen Biotech Co., Ltd.), according to the manufactuer's instructions, and cDNA was obtained using a miRcute miRNA First-Strand cDNA Synthesis kit (Tiangen Biotech Co., Ltd.). RT-qPCR was performed using a miRcute miRNA Detection kit (Tiangen Biotech Co., Ltd.). The primers for miR-497 were as follows: Forward, 5'-TCGGGCAGCAGC ACACTGTG, and universal reverse, 5'-GTGCAGGGTCCG AGGT-3'. The primers for U6 were as follows: Forward, 5'-CGC TTCGGCAGCACATATAC-3' and reverse, 5'-TTCACGAAT TTGCGTGTCAT-3'. PCR amplification was performed on an iQ5 Real-Time PCR Detection System (Bio-Rad Laboratories, Inc.), as follows: Initial denaturation at $95^{\circ} \mathrm{C}$ for $3 \mathrm{~min}$; and 40 cycles of denaturation at $95^{\circ} \mathrm{C}$ for $5 \mathrm{sec}$, and annealing at $60^{\circ} \mathrm{C}$ for $20 \mathrm{sec}$. The $2^{-\Delta \Delta \mathrm{Cq}}$ method was used to calculate the $\mathrm{miR}-497 / \mathrm{U} 6$ expression ratio.

Western blotting. Total proteins were extracted from serum and tissues using radio-immunoprecipitation assay lysis buffer (P0013B; Beyotime Institute of Biotechnology, Shanghai, China). Protein concentration was determined using a bicinchoninic acid protein concentration determination kit [cat no. RTP7102; Real-Times (Beijing) Biotechnology Co., Ltd., Beijing, China]. After boiling with loading buffer for $5 \mathrm{~min}$, protein samples $(20 \mu \mathrm{g})$ were subjected to $10 \%$ sodium dodecyl sulfate-polyacrylamide gel electrophoresis at $65 \mathrm{~V}$ until the target proteins had travelled to $1 \mathrm{~cm}$ above the edge of the gel. Next, the resolved proteins were transferred to polyvinylidene difluoride membranes on ice (100 V, 2 h) and blocked with $5 \%$ skimmed milk at room temperature for $1 \mathrm{~h}$. Subsequently, the membranes were incubated with SNCG rabbit anti-human (1:1,000; ab55424) and internal reference rabbit anti-human $\beta$-actin polyclonal primary antibodies (1:5,000; ab129348; both Abcam, Cambridge, MA, USA) at $4^{\circ} \mathrm{C}$ overnight. After extensive washing with phosphate-buffered saline with Tween-20 (CW0041; CWBIO, Beijing, China), the membranes were incubated with goat anti-rabbit horseradish peroxidase conjugated-IgG secondary antibody (1:3,000; ab6721; Abcam) 
for $1 \mathrm{~h}$ at room temperature. Then, the membrane was developed using an enhanced chemiluminescence detection kit (Sigma-Aldrich, St. Louis, MO, USA) for imaging. Image Lab software (version 3.0; Bio-Rad Laboratories, Inc.) was used to acquire and analyze imaging signals. The relative expression of SNCG protein was expressed as the ratio of SNCG to $\beta$-actin.

Statistical analysis. All statistical analyses were performed using SPSS software for Windows (version 18.0; SPSS, Inc., Chicago, IL, USA). Results are expressed as means \pm standard deviation for tests of normality. Multi-group measurements were subjected to one-way analysis of variance. In cases of homogeneity of variance, Fisher's Least Significant Difference and Student-Newman-Keuls methods were used; in cases of heterogeneity of variance, Tamhane's T2 or Dunnett's T3 methods were used. $\mathrm{P}<0.05$ was considered to indicate a statistically significant difference.

\section{Results}

SNCG $m R N A$ and protein expression levels are upregulated in osteosarcoma tissues. In order to evaluate SNCG mRNA and protein expression levels in osteosarcoma tissues, RT-qPCR and western blotting analyses were used, respectively. The RT-qPCR data revealed that mRNA expression levels in osteosarcoma tissues were significantly upregulated compared with those in adjacent tissues ( $\mathrm{P}<0.01$; Fig. 2A). In agreement, western blots indicated that SNCG protein expression levels in osteosarcoma tissues were also significantly higher compared with those of the adjacent tissues $(\mathrm{P}<0.01$; Fig. 2B). The results suggest that both $\mathrm{mRNA}$ and protein expression levels of SNCG are upregulated in osteosarcoma tissues.

SNCG $m R N A$ and protein expression levels are upregulated in the blood of patients with osteosarcoma. To examine SNCG mRNA and protein levels in the serum, RT-qPCR and western blotting analyses were performed, respectively. The RT-qPCR data revealed that SNCG mRNA levels in the serum of patients with osteosarcoma were significantly higher compared with those in healthy subjects $(\mathrm{P}<0.01$; Fig. $3 \mathrm{~A})$. In addition, western blots revealed that SNCG protein content in the serum of patients with osteosarcoma was also significantly higher compared with that in healthy subjects $(\mathrm{P}<0.05$; Fig. 3B). The results indicate that SNCG mRNA and protein levels are upregulated in the blood of patients with osteosarcoma.

SNCG $m R N A$ and protein expression levels are upregulated in lung metastatic tissues. To measure SNCG mRNA and protein expression levels in lung metastatic tissues, RT-qPCR and western blotting analyses were performed, respectively. The RT-qPCR data revealed that SNCG mRNA expression levels in lung metastatic tissues were significantly upregulated compared with those in the adjacent tissues ( $\mathrm{P}<0.01$; Fig. 4A). In addition, western blots indicated that SNCG protein expression in lung metastatic tissues was also significantly elevated compared with that in the adjacent tissues $(\mathrm{P}<0.01$; Fig. 4B). The results suggest that SNCG mRNA and protein expression levels are upregulated in lung metastatic tissues.
Downregulation of miR-497 results in the occurrence and development of osteosarcoma and its metastasis by upregulating SNCG $m R N A$. To determine the levels of miR-497 in blood, osteosarcoma and metastatic tissues, RT-qPCR was employed. The results revealed that the expression levels of miR-497 were significantly lower in osteosarcoma tissue $(\mathrm{P}<0.01$; Fig. 5A), serum $(\mathrm{P}<0.01$; Fig. 5B) and metastatic tissues $(\mathrm{P}<0.05$; Fig. $5 \mathrm{C})$. Thus, the results indicate that the downregulation of miR-497 may cause the upregulation of SNCG mRNA, resulting in the occurrence, development and metastasis of osteosarcoma.

\section{Discussion}

Osteosarcoma typically occurs in the area surrounding the knees in patients between 15 and 25 years of age, and hormone disorders during adolescence are considered to be a cause of osteosarcoma in young people( $<20$ years old) (18). Osteosarcoma can metastasize throughout the body and can be classified as one of three types: Adjacent organ invasion, implantation metastasis and distant metastasis. As a result of the abundant blood flow to the lungs and brain, the two organs are common sites for distant metastasis of osteosarcoma via the blood circulation. This can result in severe consequences, including tumors of the brain and lung $(19,20)$. Factors detectable in the blood, such as miRNA-26a, miRNA-27a and miRNA-191, may also indirectly affect the malignancy and metastasis of osteosarcoma $(21,22)$. Therefore, the elucidation of the molecular mechanisms and gene regulatory pathways involved in this process, in addition to the means to inhibit them, are important for the prevention of osteosarcoma occurrence, proliferation and invasion.

SNCG is widely distributed throughout the presynaptic region of the central nervous system, and has tissue specificity. However, SNCG is also a proto-oncogene, and its overexpression results in the interruption of mitotic checkpoints, leading to multiple nuclei in the cells. Consequently, the abnormal proliferation of these cells results in tumor formation (23). SNCG is involved in the invasion and metastasis of tumors, including those of the breast, gallbladder, pancreas and uterus (9-13), where high expression levels of SNCG have been observed. The aforementioned characteristics make SNCG a suitable tumor biomarker. The present study, which investigated SNCG levels in tumor tissues and blood samples from patients with lung metastasis of osteosarcoma, revealed abnormally upregulated SNCG expression levels. This may be important in the regulation of osteosarcoma proliferation and metastasis.

The upstream regulatory genes of SNCG were also investigated in the present study. In vertebrates, the 5'-end sequences of certain miRNAs, particularly the nucleotides at positions 2-7 (or 8), are similar. These sequences, also termed seed regions, are pairing sequences for miRNAs and their target mRNAs, and determine their functions. miRNAs that have highly similar sequences, characteristic seed regions and common target genes, are classified into a superfamily. The miR-15 superfamily is located in the 13q14 region of human chromosomes, and includes miR-15a, miR-15b, miR-16, miR-195, miR-424 and miR-497. A previous study revealed that miRNA-15a regulates the cell cycle and apoptosis of breast cancer cells through its target gene, SNCG (16). Using bioinformatic tools, the present 


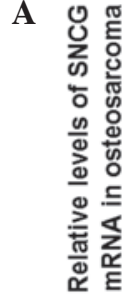

B

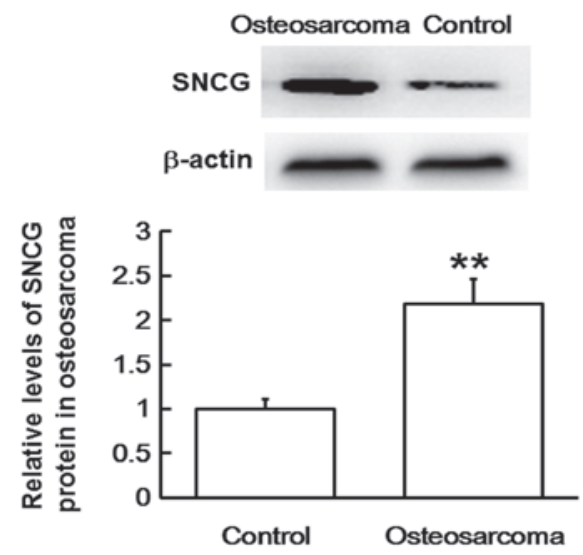

Figure 2. Expression of SNCG mRNA and protein in osteosarcoma tissues. (A) Reverse transcription-quantitative polymerase chain reaction was performed to determine relative mRNA expression levels in osteosarcoma and normal adjacent tissues. (B) Western blotting was used to measure relative protein levels of SNCG in osteosarcoma and normal adjacent tissues. Histogram data are expressed as the mean \pm standard deviation. ${ }^{* *} \mathrm{P}<0.01$ vs. control group. SNCG, synuclein $\gamma$.

A

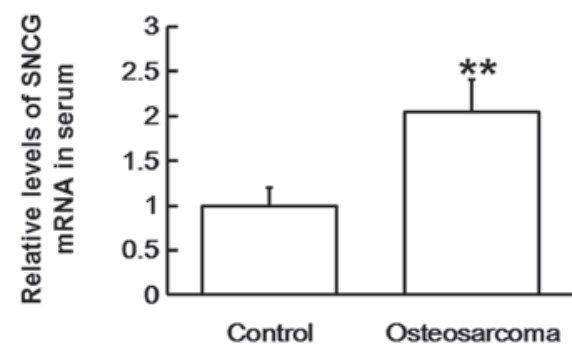

B

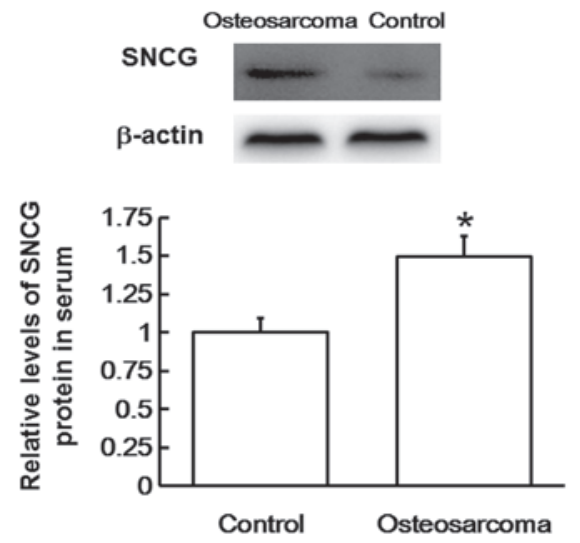

Figure 3. Expression of SNCG mRNA and protein in serum. (A) RT-qPCR was performed to determine relative mRNA expression levels of SNCG in the serum of healthy subjects and patients with osteosarcoma. (B) Western blotting was used to measure relative protein expression levels of SNCG in the serum of healthy subjects and patients with osteosarcoma. Histogram data are expressed as the mean \pm standard deviation. ${ }^{*} \mathrm{P}<0.05,{ }^{* *} \mathrm{P}<0.01$ vs. control group. SNCG, synuclein $\gamma$.
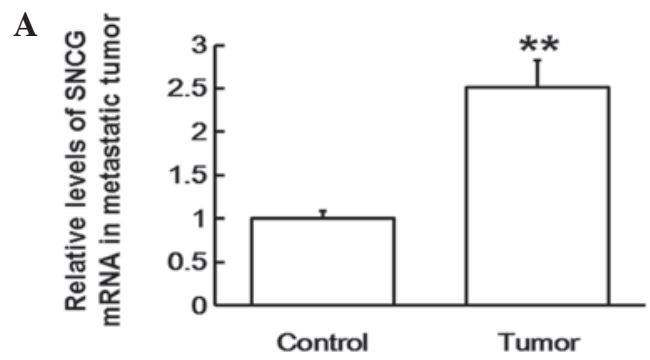

B
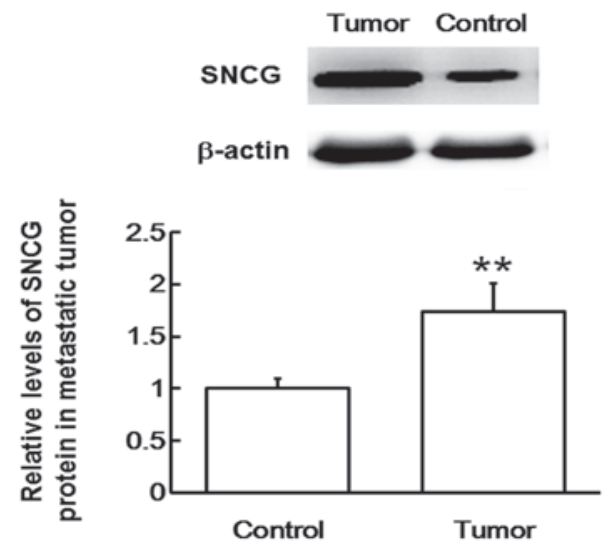

Figure 4. Expression of SNCG mRNA and protein in lung metastatic tissues. (A) RT-qPCR was performed to determine relative mRNA expression levels of SNCG in lung metastatic and normal adjacent tissues. (B) Western blotting was used to measure relative protein levels of SNCG in lung metastatic and normal adjacent tissues. Histogram data are expressed as the mean \pm standard deviation. ${ }^{* *} \mathrm{P}<0.01$ vs. control group. SNCG, synuclein $\gamma$.

study revealed that miR-497 is located within close proximity of miR-15a and possibly participates in the regulation of SNCG. Other studies have indicated that miR-497 is able to inhibit features of colon cancer by targeting insulin-like growth factor-1 receptor (24); suppress angiogenesis in ovarian cancer by targeting the vascular endothelial growth factor gene, and regulating the phosphatidylinositol 3-kinase/protein kinase B and mitogen-activated protein kinase/extracellular signal-regulated kinase signaling pathways (25); and reduce the proliferation of cervical cancer cells by targeting cyclin-E1 (26). Further studies have revealed its ability to hinder the proliferation of gastric cancer cells by targeting eukaryotic initiation factor 4E (27) and suppresses liver cancer cells by negatively regulating checkpoint kinase 1 (28). Based on these studies, we hypothesize that miR-497 may have similar regulatory effects in osteosarcoma. The current results revealed that the expression of miR-497 was reduced in blood, osteosarcoma and lung metastatic tissues, leading to a reduced regulatory effect on the tumor. This may explain the abnormal cell proliferation observed in osteosarcoma. Combining the present data on SNCG mRNA and protein expression, downregulated miR-497 may be associated with the upregulation of SNCG expression levels. The imbalance between SNCG and miR-497 may lead to the occurrence and development of osteosarcoma. Notably, the trends in expression levels of miR-497 in the blood are consistent with trends in SNCG expression levels in tumor tissues. Therefore, miR-497 in the blood may also be used for the prediction and prevention of osteosarcoma metastasis. However, a number of factors may have influenced the present study, including the limited number of patients, different surgical approaches (amputation or limb 
A

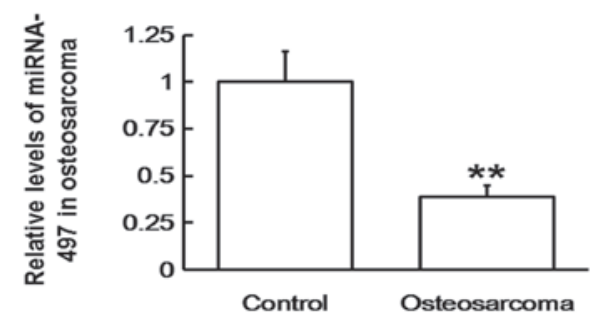

B

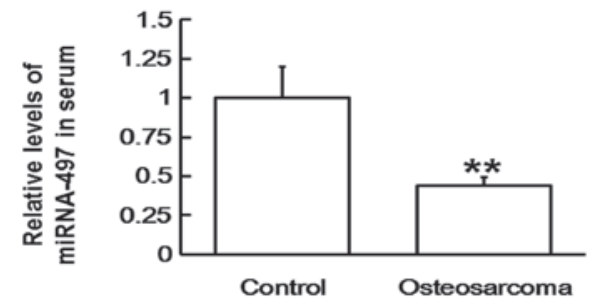

C

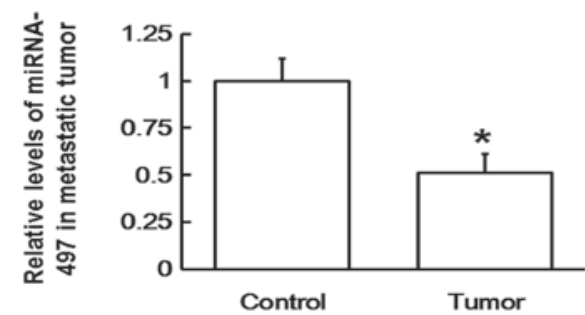

Figure 5. Expression of miR-497 in osteosarcoma. Relative expression levels of miR-497 in (A) osteosarcoma and adjacent tissues; (B) the serum of healthy subjects and patients with osteosarcoma; and (C) lung metastatic and adjacent tissues. Reverse transcription-quantitative polymerase chain reaction was performed to determine mRNA expression levels. Histogram data are expressed as the mean \pm standard deviation. ${ }^{*} \mathrm{P}<0.05,{ }^{* *} \mathrm{P}<0.01$ vs. control group. $\mathrm{miRNA} / \mathrm{miR}$, microRNA.

salvage surgery) and regions of origin, varying health conditions and complications, as well as numerous other factors associated with osteosarcoma (29-32). Therefore, further studies on cells, animals and clinical trails are necessary in order to understand the exact mechanism of action of miR-497 in osteosarcoma.

In conclusion, miR-497 may exert its inhibitory effect on the proliferation and invasion of osterosarcoma by regulating the expression levels of SNCG. As such, miR-497 may be a potential marker and therapeutic target for the treatment of osteosarcoma; however, future studies are required in order to fully elucidate the underlying mechanisms.

\section{Acknowledgements}

The present study was supported by Dr Shengtian Zhao, the director of The Second Hospital of Shandong University (Jinan, China). During the write-up, we received help and constructive suggestions from Dr Wenguang Liu and Dr Haipeng Si of Shandong University.

\section{References}

1. Lei P, Xie J, Wang L, Yang X, Dai Z and Hu Y: microRNA-145 inhibits osteosarcoma cell proliferation and invasion by targeting ROCK1. Mol Med Rep 10: 155-160, 2014

2. Jaffe N. Osteosarcoma: Review of the past, impact on the future. The American experience. Cancer Treat Res 152: 239-262, 2009

3. Ferguson WS and Goorin AM. Current treatment of osteosarcoma. Cancer Invest 19: 292-315, 2001.
4. Hameed $M$ and Dorfman H. Primary malignant bone tumours - recent developments. Semin Diagn Pathol 28: 86-101, 2011.

5. Li R, Liu J, Wu H, Liu L, Wang L and Zhang S: TIKI2 suppresses growth of osteosarcoma by targeting Wnt/ $\beta$-catenin pathway. Mol Cell Biochem 392: 109-116, 2014.

6. Mao JH, Zhou RP, Peng AF, Liu ZL, Huang SH, Long XH and Shu Y: microRNA-195 suppresses osteosarcoma cell invasion and migration in vitro by targeting FASN. Oncol Lett 4: 1125-1129, 2012.

7. Jiang Y, Liu YE, Goldberg ID and Shi YE: Gamma synuclein, a novel heat-shock protein-associated chaperone, stimulates ligand-dependent estrogen receptor alpha signaling and mammary tumorigenesis. Cancer Res 64: 4539-4546, 2004.

8. Ji H, Liu YE, Jia T, Wang M, Liu J, Xiao G, Joseph BK, Rosen C and Shi YE: Identification of a breast cancer-specific gene, BCSG1, by direct differential cDNA sequencing. Cancer Res 57: 759-764, 1997.

9. Morgan J, Hoekstra AV, Chapman-Davis E, Hardt JL, Kim JJ and Buttin BM: Synuclein- $\gamma$ (SNCG) may be a novel prognostic biomarker in uterine papillary serous carcinoma. Gynecol Oncol 114: 293-298, 2009.

10. Hibi T, Mori T, Fukuma M, Yamazaki K, Hashiguchi A, Yamada T, Tanabe M, Aiura K, Kawakami T, Ogiwara A, et al: Synuclein- $\gamma$ is closely involved in perineural invasion and distant metastasis in mouse models and is a novel prognostic factor in pancreatic cancer. Clin Cancer Res 15: 2864-2871, 2009.

11. Han S, She F, Wang D, Yao X, Jiang L and Chen Y: SNCG gene silencing in gallbladder cancer cells inhibits key tumorigenic activities. Front Biosci (Landmark Ed) 17: 1589-1598, 2012.

12. Pan ZZ, Bruening W, Giasson BI, Lee VM and Godwin AK: Gamma-synuclein promotes cancer cell survival and inhibits stress- and chemotherapy drug-induced apoptosis by modulating MAPK pathways. J Biol Chem 277: 35050-35060, 2002.

13. Liu H, Liu W, Wu Y, Zhou Y, Xue R, Luo C, Wang L, Zhao W, Jiang JD and Liu J: Loss of epigenetic control of synuclein- $\gamma$ gene as a molecular indicator of metastasis in a wide range of human cancers. Cancer Res 65: 7635-7643, 2005.

14. Dorfman HD, Czemiak B and Kotz R: World Health Organization Classification of Tumours. In: World Health Organization Classification of Tumours, Pathology and Genetics of Tumours of Soft Tissue and Bone. Fletcher CDM, Unni KK and Mertens F (eds). IARC Press, Lyon, France, pp227-232, 2002.

15. Enneking WF (ed): Staging musculoskeletal tumors. In: Musculoskeletal Tumor Surgery. Churchill Livingstone, New York, pp69-122, 1983.

16. Li P, Xie XB, Chen Q, Pang GL, Luo W, Tu JC, Zheng F, Liu SM, Han L, Zhang JK, et al: MiRNA-15a mediates cell cycle arrest and potentiates apoptosis in breast cancer cells by targeting synuclein-gamma. Asian Pac J Cancer Prev 15: 6949-6954, 2014.

17. Livak KJ and Schmittgen TD: Analysis of relative gene expression data using real-time quantitative PCR and the $2-\Delta \Delta \mathrm{Ct}$ method. Methods 25: 402-408, 2001.

18. Zhou X, Jing J, Peng J, Mao W, Zheng Y, Wang D, Wang X, Liu Z and Zhang X: Expression and clinical significance of galectin-3 in osteosarcoma. Gene 546: 403-407, 2014.

19. Shweikeh F, Bukavina L, Saeed K, Sarkis R, Suneja A, Sweiss F and Drazin D: Brain metastasis in bone and soft tissue cancers: A review of incidence, interventions, and outcomes. Sarcoma 2014: 475175, 2014.

20. Link MP, Gebhardt MC, Meyers PA. Chapter 35: Osteosarcoma. In: Principles and Practice of Pediatric Oncology. Pizzo PA and Poplack DG (eds). 5th edition. Lippincott Williams \& Wilkins Philadelphia, PA, pp1051-1089, 2006.

21. Taheriazam A, Bahador R, Karbasy SH, Jamshidl SM, Torkaman A, Yahaghi E and Shakeri M. Down-regulation of microRNA-26a and up-regulation of microRNA-27a contributes to aggressive progression of osteosarcoma. Diagn Pathol 10: 166, 2015.

22. Wang T, Ji F, Dai Z, Xie Y and Yuan D. Increased expression of microRNA-191 as a potential serum biomarker for diagnosis and prognosis in human osteosarcoma. Cancer Biomark 15: 543-550, 2015.

23. Ninkina NN and Bukhman VL. Synucleins - to have or not to have. Genetika 36: 1487-1491, 2000 (In Russian).

24. Guo ST, Jiang CC, Wang GP, Li YP, Wang CY, Guo XY, Yang RH, Feng Y, Wang FH, Tseng HY, et al: MicroRNA-497 targets insulin-like growth factor 1 receptor and has a tumour suppressive role in human colorectal cancer. Oncogene 32: 1910-1920, 2012. 
25. Wang W, Ren F, Wu Q, Jiang D, Li H and Shi H: MicroRNA-497 suppresses angiogenesis by targeting vascular endothelial growth factor A through the PI3K/AKT and MAPK/ERK pathways in ovarian cancer. Oncol Rep 32: 2127-2133, 2014.

26. Han J, Huo M, Mu M, Liu J and Zhang J: miR-497 suppresses proliferation of human cervical carcinoma HeLa cells by targeting cyclin E1. Xi Bao Yu Fen Zi Mian Yi Xue Za Zhi 30: 597-600, 2014 (In Chinese).

27. Li W, Jin X, Deng X, Zhang G, Zhang B and Ma L: The putative tumor suppressor microRNA-497 modulates gastric cancer cell proliferation and invasion by repressing eIF4E. Biochem Biophys Res Commun 449: 235-240, 2014.

28. Xie Y, Wei RR, Huang GL, Zhang MY, Yuan YF and Wang HY: Checkpoint kinase 1 is negatively regulated by miR-497 in hepatocellular carcinoma. Medical Oncology 31: 844, 2014.

29. Ohba T, Cates JM, Cole HA, Slosky DA, Haro H, Ando T, Schwartz HS, Schoenecker JG: Autocrine VEGF/VEGFR1 Signaling in a Subpopulation of Cells Associates with Aggressive Osteosarcoma. Molecular Cancer Research molcanres. 0037.2014, 2014.
30. Liu H, Huang L, Zhang Z, Zhang Z, Yu Z, Chen X, Chen Z, Zen Y, Yang D, Han Z, et al: LIM mineralization protein-1 inhibits the malignant phenotypes of human osteosarcoma cells. International journal of molecular sciences 15: 7037-7048, 2014.

31. He H, Ni J and Huang J: Molecular mechanisms of chemoresistance in osteosarcoma. Oncol Lett 7: 1352-1362, 2014 (Review).

32. Zhang H, Yin Z, Ning K, Wang L, Guo R and Ji Z: Prognostic value of microRNA-223/epithelial cell transforming sequence 2 signaling in patients with osteosarcoma. Hum Pathol 45: 1430-1436, 2014. 